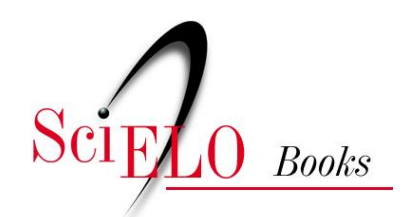

\title{
Seduepb
}

\section{Re-construcción de la cultura y del espacio turístico}

\author{
Julio César C. Medina
}

\section{SciELO Books / SciELO Livros / SciELO Libros}

MEDINA, JC. Re-construcción de la cultura y del espacio turístico. In BRASILEIRO, MDS., MEDINA, JCC., and CORIOLANO, LN., orgs. Turismo, cultura e desenvolvimento [online]. Campina Grande: EDUEPB, 2012. pp. 21-47. ISBN 978-85-7879-194-0. Available from SciELO Books < http://books.scielo.org>.

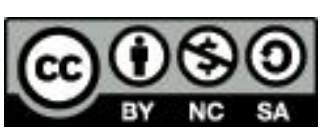

All the contents of this work, except where otherwise noted, is licensed under a Creative Commons Attribution-Non Commercial-ShareAlike 3.0 Unported.

Todo o conteúdo deste trabalho, exceto quando houver ressalva, é publicado sob a licença Creative Commons Atribuição Uso Não Comercial - Partilha nos Mesmos Termos 3.0 Não adaptada.

Todo el contenido de esta obra, excepto donde se indique lo contrario, está bajo licencia de la licencia Creative Commons Reconocimento-NoComercial-CompartirIgual 3.0 Unported. 
Parte I

Turismo e Desenvolvimento 



\title{
Re-construcción de la cultura y del espacio turístico
}

\author{
Julio César C. Medina
}

\section{Introducción}

Las teorías sobre el turismo contribuyen a la comprensión y/o explicación del fenómeno del turismo. La compresión nos informa de por qué los agentes realizan sus acciones, esto es los fundamentos de las acciones. La explicación nos informa de qué es lo que los agentes producen con sus acciones, es decir, el resultado de sus acciones. Sin embargo, como nos advierte Feyerabend (1993), las teorías sociales no pueden ser consideradas una representación fiel de la realidad. Toda investigación científica sobre el turismo se inicia por la definición del objeto de investigación y esto supone elegir algunos aspectos de la realidad y excluir otros. Este acto de categorizar la realidad es un acto de cirugía para 
sistematizar el pensamiento (teoría) y los procedimientos para comprenderlos (metodología). Bauman considera que:

Determinadas entidades pueden ser incluidas en una clase - hechas una clasesólo en la misma proporción en que otras entidades son excluidas, apartadas. Invariablemente, semejante operación de inclusión/exclusión es un acto de violencia perpetrado al mundo y requiere el soporte de una cierta coerción" (BAUMAN, 1996, p. 75).

Toda teoría es parcial y provisional, y sólo por cuestiones prácticas y metodológicas damos por concluida en una primera fase de la investigación. Las categorías son parciales puesto que recogen parte de la realidad y no la totalidad del fenómeno estudiado. Posteriormente, las categorías deben ser sometidas a prueba mediante la investigación científica, y en este sentido son provisionales para ser reformuladas y aplicadas a otros objetos de investigación posteriormente. Es conveniente pues, recordar con Einstein que "el hombre procura formarse una imagen adecuada y fácilmente aprehensible del mundo, con el fin de sobreponerla a la experiencia de la realidad, sustituyéndola hasta cierto grado por ella" (EINSTEIN, 1986, p. 147).

Esta propuesta que aquí presentamos no pretende ser una representación fiel dela realidad, sino una construcción teórica que intentan abordar algunos aspectos de la complejidad del fenómeno del turismo desde una perspectiva constructivista. El constructivismo que defendemos intenta integrar distintas perspectivas que nos permitan analizar la realidad material y 
simbólica y sus efectos en la re-construcción cultural y espacial del fenómeno del turismo. Previamente a estas cuestiones, nuestro análisis comienza con una reflexión epistemológica del turismo, para seguidamente abordar el turismo como un conjunto de realidades múltiples donde distintos agentes participan de dicho proceso de re-construcción del turismo.

\section{La complejidad epistémica del fenómeno turístico}

Elanálisisdeldesarrolloturísticoseencuentracondicionado por la representación negativa que este fenómeno social tiene entre los teóricos sociales que teorizan y analizan el desarrollo. La imagen más difundida del turismo es la de un fenómeno de masas, una horda dorada que destruye el medio ambiental, mercantiliza las culturas locales, somete a los trabajadores locales a trabajos denigrantes o mal remunerados y coloniza o destruye el tejido social de las comunidades donde se asienta. Como ya fue expresado por J. Krippendorf (2003) a pesar de que casi todos nosotros viajamos, con mayor o menor frecuencia, sería difícil encontrar personas más criticadas, acusadas, ridiculizadas e insultadas que los turistas. El predominio del paradigma economicista ha sido la una de las causas de la falta de interés académico por abordar el fenómeno del turismo de forma más compleja y la falta de iniciativas políticas que relacionen esta actividad con el desarrollo socio-económico-cultural de las localidades.

El paradigma científico dominante en gran parte del siglo XX destacaba el concepto de trabajo y la producción como elementos centrales, descuidando otras dimensiones de la realidad. El estudio de Weber (1998) sobre la ética 
protestante y el espíritu del capitalismo intenta responder a la pregunta: ¿qué fenómenos culturales contribuyeron para que el capitalismo se desarrollase en Occidente y no en otras partes del mundo? La respuesta que Weber ofreció es que los valores morales juegan un papel importante en el proceso del desarrollo socio-económico. A partir de esta obra se inició un profundo debate entre los defensores del materialismo y los defensores del idealismo sobre la compresión y/o explicación del proceso de re-construcción social de la realidad. No obstante, conviene recordar las palabras que encierran el análisis de Weber:

Nuestra intención no es tampoco sustituir una concepción unilateralmente 'materialista' de la cultura y de la historia por una concepción contraria de unilateral causalismo espiritual. Materialismo y espiritualismoson interpretaciones posibles, pero como trabajo preliminar; si, por el contrario, pretenden constituir el término de la investigación, ambas son igualmente inadecuadas para servir la verdad histórica (WEBER, 1998, p. 226-227).

La finalidad de nuestra propuesta consiste en ofrecer un análisis del fenómeno del turismo desde una perspectiva constructivista que integre la dimensión material y la dimensión simbólica de la realidad (BERGER, LUCKMANN, 1994). El turismo es un proceso complejo caracterizado por la continua re-construcción material y simbólica, concretizados por agentes sociales que actúan en contextos sociales estructurados (normas, reglas, etc.), utilizando una diversidad de recursos 
a partir de interpretaciones de la realidad que también son socialmente construidas. Para evitar un análisis que sólo contemple una de estas dos dimensiones del turismo, adoptamos el concepto de complejidad expresado por Morin:

A primera vista la complejidad es un tejido (complexus: que está tejido en conjunto) de constituyentes heterogéneos inseparablemente asociados: presenta la paradoja de lo uno y de lo múltiple. Al mirar con más atención, la complejidad es, efectivamente, el tejido de eventos, acciones, interacciones, retroacciones, determinaciones, azares que constituyen nuestro mundo fenoménico (MORIN, 1996, p. 32).

En este sentido, podemos considerar el fenómeno del turismo como una realidad compleja desde un doble punto de vista: por un lado, el turismo está constituido por una complejidad sistémica (diversidad de agentes) y una complejidad fenoménica (diversidad de interpretaciones), que veremos a continuación. Y por otro lado, el turismo presenta una dimensión simbólica expresada en distintas interpretaciones socialmente construidas sobre la realidad material, y a la vez, una dimensión material, en forma de productos sedimentados en los espacios turísticos, que fueron construidos a partir de las interpretaciones de distintos agentes a lo largo de la historia.

Una de las características fundamentales del proceso de re-construcción del turismo es su complejidad sistémica, entendida comola sobreabundancia de relaciones y conexiones 
entre los elementos de un sistema, de modo que ya no es posible la relación unívoca y lineal entre sus partes. Luhmann (1997) considera que "al aumentar el número de elementos que deben englobarse en un sistema o para un sistema en tanto que su entorno, muy pronto se alcanza un punto a partir del cual se hace imposible que cada elemento se relacione con todos los demás" (LUHMANN, 1997, p. 68-69). En el fenómeno del turismo, esta complejidad sistémica se puede observa en la diversidad de agentes que participan del proceso: agentes globales como las instituciones internacionales (Unesco, Organización Mundial del Turismo, etc.) instituciones federales, estatales y locales. A modo de ejemplo, la UNESCO normalmente no se relaciona directamente con la población local o con los turistas que visitan una localidad, pero su decisión de catalogar un lugar como Patrimonio de la Humanidad (re-construcción simbólica) puede favorecer el desarrollo turístico, contribuyendo así, en el proceso de construcción del espacio turístico de esa localidad (re-construcción material).

La complejidad fenoménica del turismo hace referencia a la diversidad de interpretaciones de los agentes que actúan en una localidad. Los agentes posen un conjunto de significados culturalmente construidos y diferencialmente distribuidos. Los agentes re-construyen su interpretación de la realidad a partir de las acciones realizadas en el pasado y contrastándolas con la situación actual. El conocimiento de cada uno de los agentes sobre la realidad está condicionado por su historia de acciones y por los distintos tipos de conocimiento sobre ella. Además, para construir su interpretación de la realidad, los agentes atribuyen sentidos a 
las acciones de otros agentes y a las condiciones del contexto donde actúan. Habermas así lo expresa:

La complejidad (...) que acosa a los sistemas sociales, no es la complejidad de la realidad sino la del 'mundo' construido por vía de sentido, que genera los propios sistemas sociales como entorno de ellos por la vía de la aprehensión simbólicamente mediada de la complejidad del mundo (HABERMAS, 1996, p. 320).

Los distintos agentes observan la misma realidad, pero construyen interpretaciones diferentes, debido a que ocupan posiciones diferentes. Comprender las interpretaciones de los distintos agentes, y de las posibles diferencias de interpretación en el interior de cada uno de estos agentes es de vital importancia para analizar el turismo de forma compleja. Por ejemplo, dentro de la categoría instituciones públicas, podemos encontrar instancias que defienden la conservación de la estructura urbana y/o de las tradiciones culturales, así como aquellas que defienden la necesidad de modificar las estructuras urbanas y promover el desarrollo económico y la interacción cultural para el desarrollo turístico.

El proceso de re-construcción del turismo requiere de una profundareflexión epistemológicaque no es posible desarrollar aquí, pues no es el objetivo de este ensayo (MEDINA, 2005). No obstante, y de forma sintética, hemos destacado que la realidad social, y por extensión el fenómeno del turismo, es una realidad compleja que presenta una dimensión material/ simbólica y una complejidad sistémica/fenoménica. 


\section{El turismo como conjunto de realidades múltiples}

El turismo es un conjunto de realidades múltiples, producto de las acciones de agentes que participaron en el pasado y de agentes que participan en el presente en la re-construcción del fenómeno turístico. El concepto de re-construcción aquí utilizado se refiere tanto a los resultados concretos de las acciones (explicación) como a los fundamentos simbólicos de las acciones (comprensión). Los productos materiales y simbólicos construidos por el turismo son resultado da las acciones de múltiples agentes que no necesariamente obedecen a un plano predeterminado de actuación conjunta. Los distintos agentes participan de forma diferenciada en función de los recursos culturales y materiales disponibles y, también, en función de los distintos contextos en los que actúan. Santos (2007) considera que el espacio social hay que entenderlo como una unidad o totalidad que se transforma de acuerdo a los cambios de la sociedad, por una diversidad de agentes en escalas diferentes:

La producción del espacio es el resultado de múltiples determinaciones, cuyo origen se sitúa en niveles diferentes y escalas variables, yendo del simple lugar a la dimensión internacional. (SANTOS, 2007, p. 58-59).

Para entender la cuestión de las realidades múltiples ya nos hemos referido al efecto que la UNESCO puede tener en una localidad específica. Volviendo a tomar este ejemplo, desde el punto de vista del espacio físico objeto de intervención, la UNESCO y las instituciones públicas locales actúan sobre 
el mismo espacio. Sin embargo, no es el mismo espacio desde el punto de vista del significado atribuida al espacio, ni de los recursos que cada uno posee para modificarlo. Las instituciones públicas locales se encuentra situadas en el espacio y la UNESCO interfiere en este espacio sin estar situado en el mismo. Así, el turismo es un conjunto de agentes que tienen distintas interpretaciones de la realidad, que poseen diferentes recursos y que están situados en distintas parcelas de la realidad que forman la unidad. Esto es lo que Morin denomina la paradoja de lo uno y lo múltiple (MORIN, 1996).

Los agentes que participan del proceso de re-construcción del turismo tienen un conocimiento limitado, a partir del cual representan su realidad y ejecutan sus acciones. Esta cuestión es importante, para evitar depositar exceso de expectativas en planes y programas de desarrollo que no abordan una perspectiva compleja para comprender e intervenir en el turismo. March y Simon (1993) consideran que los diferentes actores presentan una racionalidad limitada de la realidad, es decir, escogen sus comportamientos según sus visiones parciales y simplificadas. Estos autores afirmarán que:

Debido a los límites de las capacidades humanas en comparación con las complejidades de los problemas que se presentan a los individuos y las organizaciones, un comportamiento racional requiere modelos simplificados que capten los aspectos principales de un problema sin captar todas sus complejidades. (MARCH \& SIMON, 1993, p. 379). 
Comprender el proceso de re-construcción del turismo requiere incorporar la interpretación de los distintos agentes, sus marcos de referencias, sus objetivos, las estrategias elaboradas y las acciones realizadas, esto es, comprender la racionalidad limitada de cada uno de ellos. Así, la elaboración de un plan de desarrollo turístico requiere incluir la mayor información posible en el diagnóstico de la realidad para trazar las estrategias, programas, proyectos e intervenciones específicas. Las interpretaciones de los distintos agentes son un elemento clave y debe ser incluido en dicho diagnóstico. Sin embargo, esto no significa que la representación social de cada uno de los agentes puedan ser consideradas estáticas. La realidad social es procesual, debido a que los agentes son reflexivos, modificando sus interpretaciones de la realidad y, consecuentemente, sus acciones. S. Lash considera que la reflexividad puede ser entendida de dos formas:

La primera es que existe una reflexividad estructural en la que la agencia, liberada de las constricciones de la estructura social, reflexiona las 'reglas' y 'recursos' de tal estructura; reflexiona sobre las condiciones sociales de existencia de la agencia. La segunda es que existe una auto-reflexividad en la que la agencia reflexiona sobre sí misma. (LASH, 1996, p. 143-144).

De esta forma, entendemos que los agentes que participan de un proceso de desarrollo turístico reflexionan sobre el contexto, los recursos, normas y reglas que delimitan sus acciones; como también reflexionan sobre sus marcos 
de referencias para el análisis del contexto, a partir de las interpretaciones que hacen de las estrategias de los otros agentes, re-defiendo su representación del contexto y re-definiendo sus estrategias y objetivos. Este carácter reflexivo de la realidad nos permite analizar el carácter dinámico, los cambios en el contexto y los cambios en las interpretaciones del contexto de cada uno de los agentes, en una unidad mayor caracterizada por ser una realidad múltiple.

\section{El turismo y la re-construcción simbólica de la cultura}

El proceso de colonización de occidente en el siglo XIX, originó un proceso de hibridación cultural en un doble sentido: de un lado, el éxodo rural que no encontró trabajo en las ciudades europeas provoco la inmigración hacia las colonias produciendo el proceso de occidentalización del mundo (HOSBAWM, 2005). Por otro lado, el tráfico de esclavos procedente de África hacia América y Europa originó una africanización en las regiones receptoras de esclavos, dándose un proceso de hibridación cultura entre nativos, europeos y africanos. Ahora bien, si estos procesos migratorios significaron una importante transformación cultural en las metrópolis y en las colonias del siglo XIX, ¿cuál es el efecto del turismo sobre el proceso de hibridación cultural en la actualidad?

El proceso de globalización se extiende en el tiempo desde la época de las grandes navegaciones, como sostiene Wallerstein (2002), y se extiende también en el espacio, a partir de las redes de la información como defiende Castells (2005). Pero también, adquiere una alta densidad, 
transformando las culturas nacionales, por efecto de los espacios sociales transnacionales como afirma Beck (1999). La globalización no es una novedad de inicios del siglo XXI y produce profundas transformaciones espaciales y culturales en las localidades. Nada mejor que recordar las palabras de Marx (1977) para sintetizar estos procesos:

Ya no reina aquel mercado local y nacional que se bastaba a satisfacer a sí mismo y donde no entraba nada de fuera; a hora, la red del comercio es universal y en ella entran, unidas por vínculos de interdependencia, todas las naciones. Y lo que acontece con la producción material, acontece también con la del espíritu. Los productos espirituales de las diferentes naciones vienen a formar un acervo común. Las limitaciones y peculiaridades del carácter nacional van pasando a un segundo plano, y las literaturas locales y nacionales confluyen todas en una literatura universal. (MARX, 1977, p. 27).

En la sociedad postindustrial de la actualidad, el turismo adquiere una importancia fundamental, tanto desde el punto cuantitativo, (número de recursos humanos, financieros infraestructura y de desplazamientos de turistas) como desde el punto de vista cualitativo, al revalorizar simbólicamente algunos espacios urbanos, espacios naturales, edificios emblemáticos, monumentos y/o prácticas socio-culturales (MEDINA, 2009). Desde el punto de la re-construcción simbólica se producen dos consecuencias importantes: de un lado, una re-construcción de la historia local para ser ofrecidos 
como productos turísticos, sea en forma de patrimonio arquitectónico, o sea en forma de rituales/costumbres que son redefinidos en las localidades. Por otro lado, esta re-definición de los elementos emblemáticos de la historia local afecta a la re-definición de las identidades locales como sostenemos a continuación.

La globalización significa un proceso de homogenización y, al mismo tiempo, un proceso de heterogenización cultural (FEATHERSTONE, 1996). Para este autor, el mundo no está viviendo un empobrecimiento cultural, una reducción de los recursos culturales, sino que, y simultáneamente, los medios de comunicación y las nuevas tecnologías de la comunicación favorecen el intercambio cultural. La forma en que las localidades se adaptan a estos procesos es de mayor complejidad de la apuntada por los defensores de la homogenización cultural. Como ejemplo, este autor expone que los Inuit de Alaska reciben turistas para participar de su vida tribal, bajo supervisión de agencias institucionales. Los recursos económicos obtenidos son usados para comprar equipamientos (rifles y municiones) y mantener su vida de cazadores parcialmente modernizada y vendida a segmentos específicos de turistas. En palabras de Featherstone: los Inuit "están en situación de poseer recursos de poder suficiente para manipular las fronteras de su comunidad para sus propios fines y mantener su identidad cultural" (FEATHERSTONE, 1996, p. 33).

El turismo contribuye a la re-construcción de las identidades culturales de forma compleja. Para entender mejor este proceso podemos utilizar la tipología ofrecida por Hall (2006) para el análisis del proceso de globalización 
sobre las identidades: por un lado, se produce un proceso de desintegración de algunos elementos de la cultura; por otro lado, un proceso de reforzamiento de otros elementos, como forma de resistencia a los procesos globales. Y por último, un proceso de hibridación o combinación de elementos culturales que dan origen nuevas formas culturales. En el caso del turismo, podemos analizar cómo algunos elementos de las culturas son desintegrados por el efecto de determinado tipo de turismo como, por ejemplo, el turismo de masa (BOYER, 2003; KRIPPENDORF, 2003). Otros elementos culturales son reforzados y revitalizados para entrar en el abanico de productos culturales ofrecidos al turismo por efecto del turismo cultural (HARVEY, 2006). Por último, otros elementos culturales son refundidos o hibridados por efecto de la interacción entre los turistas y los residentes (CANCLINI, 2006; HIERNAUX, 2000).

El turismo no solo consume espacios naturales (sol y playa, montaña, nieve, etc.). o espacios urbanos, sino que también consume tiempos históricos. El espacio construido es un resumen de la historia pasada que puede ser visitado en el presente. Según Bauman (2002), asistimos a una época de re-ciclaje, donde nada parece morir del todo y todo es $r e$-inventado, $r e$-pensado y re-ciclado, para ser $r e$-introducido como tradicional. Ante los cambios de los marcos de referencia culturales en la sociedad post-moderna, el turismo cultural participa del proceso de re-construcción de la realidad local, al demandar espacios de alto contenido histórico-simbólico. A su vez, agentes locales defienden el patrimonio histórico como forma de reforzar la identidad local frente al proceso de globalización cultural. Lo que puede ser entendido como un proceso de mercantilización de la cultura y de la historia 
para uso turístico (HARVEY, 2006) puede significar también un proceso de re-construcción y fortalecimiento de las identidades. Los agentes locales entablan una lucha por la definición de lo que debe ser preservado, por la re-construcción y preservación de la historia, en definitiva, por la re-construcción de los elementos esenciales de su identidad histórica por efecto del fenómeno del turismo.

\section{El turismo y la re-construcción material del espacio}

El proceso de re-construcción material del espacio turístico puede ser entendido a partir de los conceptos de sistema de lugares funcionales y grupo geográfico-sociales de comportamiento. El análisis los lugares funcionales nos permite explicar/comprender cómo se estructura el espacio para satisfacer las necesidades sociales, como se producen cambios en la forma de uso del territorio y, específicamente, los usos turísticos. Para Maier (1987) un sistema espacial está formado por el conjunto de todas las informaciones existente sobre el espacio social. Estas informaciones son organizadas por criterios de preferencia espaciales de los distintos agentes que motivaran las decisiones y sus comportamientos. Las preferencias espaciales se manifestarán a través las actividades de trascendencia espacial, en el marco de las funciones fundamentales (vivienda, abastecimiento, trabajo, educación y ocio). La concentración espacial de estas funciones determinará la estructura socio-espacial en forma de lugares funcionales. Por último, los cambios en las preferencias y las actividades de transcendencia espacial producen cambios en el sistema de lugares funcionales. 
Lefebvre (1976) propone analizar el espacio a partir de dos categorías que superen las limitaciones del funcionalismo urbanístico y que adaptamos para analizar el uso turístico del espacio: en primer lugar, el espacio es multi-funcional, o sea, un mismo espacio puede satisfacer varias necesidades y desempeñar distintas funciones. Por ejemplo, el turismo cultural puede utilizar espacios, que dentro del sistema de lugares funcionales, obedecen a otras funciones sociales para la comunidad residente. En este sentido seria un espacio multi-funcional, es decir, espacio comercial de día, bares y restaurantes de noche, etc. En segundo lugar, el espacio es trans-funcional, esto es, el espacio social es soporte de una o varias funciones sociales, pero también puede ser analizado como espacio donde se desarrollan actividades afectivas, relacionales y lúdicas de la sociedad. Por ejemplo, podemos considera un espacio determinado como lugar funcional de ocio por la concentración de actividades de este tipo. Ahora bien, las actividades de ocio pueden ser consideradas una forma de reforzarlas relaciones sociales, laidentidad local, las prácticas de convivencialidad (ILLICH, 1974), de solidaridad, de ayuda mutua, o como define Maffesoli (1990) "estar juntos sin ocupación", sin intereses instrumentales que definan la interacción, pero que no por eso dejan de tener consecuencias en la re-construcción del espacio.

El proceso de re-construcción del espacio turístico es realizado por agentes que realizan actividades de trascendencia espacial. Entenderemos las actividades de transcendencia espacial turísticas como aquellas actividades que afectan a la re-construcción del espacio de uso turístico de forma efectiva. 
Así, entenderemos como agentes, los grupos geográficosociales de comportamiento, de la siguiente forma:

Cuando un cierto número de hombres se hallan en una situación social comparable y, de acuerdo con ello, desarrollan modos de comportamiento con una influencia similar en los procesos y estructuras espaciales, entonces se puede incluir a estos hombres en el mismo 'grupo geográfico-social de comportamiento’ (MAIER, 1987, p. 36-54).

De esta forma, podemos establecer una relación entre la complejidad sistémica de los lugares funcionales, con la complejidad fenoménica de los distintos agentes que participan en la re-construcción espacial de uso turístico. Es decir, debemos especificar características comunes que definan a los distintos agentes, así como determinar las preferencias espaciales, actividades de transcendencia espaciales y los resultados de las acciones de cada uno de estos agentes, en la re-construcción de lugares funcionales de uso turístico. Como ya hemos indicado con anterioridad, podemos identificar como agentes a las instituciones internacionales, instituciones públicas, empresas, movimientos sociales, residentes y turistas. Ahora bien, para cada objeto de investigación concreto debemos determinar esa 'situación social comparable' y eses 'modos de comportamiento' que los identifiquen como grupos geográfico-sociales de comportamiento, como sugiere Maier (1987). Para comprender esta cuestión proponemos, 
analizar los agentes y sus modos de comportamientos en centros urbanos históricos (PABLOS; MEDINA: 2004):

1. Algunos movimientos sociales tienen como objetivo esencial la preservación del patrimonio y realizan acciones para delimitar simbólicamente lo que debe y no debe ser conservado, lo que podemos clasificar como lógica conservacionista. A su vez, otros movimientos sociales instaban a las instituciones públicas a desarrollar planes y programas de conservación, con el mayor grado de participación posible, dentro de una lógica que hemos denominado de lógica de la participación.

2. Las instituciones públicas realizan planes y programas que deben estar ajustadas a las normas legales dentro de la lógica burocrática. Algunas de las iniciativas políticas tuvieron éxito y fomentaron la inversión de empresas vinculadas al turismo, que actuarán con la finalidad de obtención de lucro, que hemos llamado de lógica mercantil.

3. El aumento de la actividad turística fomenta la interacción entre los distintos tipos de residentes, especialmente con aquellos que trabajan en el sector turístico, provocando la lógica del encuentro entre ellos. Mientras tanto, dentro del grupo de residentes podemos encontrar otros grupos que rechazan la interacción con los turistas, produciendo la lógica del des-encuentro.

4. Por efecto del uso turístico multi-funcionalidad del espacio, algunos residentes mudaron sus preferencias socio-espaciales en relación a la residencia y/o en relación a los espacios de ocio (trans-funcionalidad) 
y modificaron sus actividades de transcendencia espacial. Estos cambios en las preferencia y en las acciones de transcendencia espacial se concretizaron en cambios en el sistema de lugares funcionales.

5. En resume, el análisis del fenómeno del turismo necesita incorporar el mayor grado de complejidad posible para cada objeto y para cada investigación específica e identificar las formas de participación en la re-construcción del espacio turístico para cada agente.

El espacio turístico es el resultado de las distintas lógicas y de las relaciones de cooperación y de conflicto de los agentes que participan en la re-construcción del espacio (CORIOLANO, 2005). En la teoría funcionalista clásica, el conflicto es considerado como disfuncional o patológico, al considerar que es resultado del deterioro de las normas y reglas existentes. En formulaciones más avanzadas del funcionalismo, como Darhendorf (1979), el conflicto presenta un carácter funcional, al fortalecer la integración de los individuos que pertenecen a un colectivo y se definen en oposición a otro grupo. Además, la sociedad actual se caracteriza por la institucionalización del conflicto, motivado por la necesidad que sienten los agentes de resolver problemas de cooperación y de acción conjunta, como puede ser el desarrollo de un plan de desarrollo turístico.

Por su parte, el materialismo histórico ha considerado el conflicto como elemento integrante y organizador de la sociedad, en el sentido de contribuir en el proceso de re-construcción de la sociedad. El conflicto no sólo no es dis-funcional, sino que es una dinámica esencial para la comprensión del fenómeno del turismo. El conflicto pude 
ser considerado como el antagonismo entre varios agentes con intereses contrarios, en cuanto a la posesión de recursos materiales y/o simbólicos, en el proceso de re-construcción del espacio turístico.

El análisis de los recursos turísticos, frecuentemente se dirige hacia la definición de los recursos naturales, históricosculturales, manifestaciones populares u otros. Si bien esta clasificación puede resultar útil para la planificación del turismo, precisamos de una visión más compleja del concepto de recursos y encuadrarla dentro de las relaciones de poder que actúan en el proceso de re-construcción del espacio turístico. El análisis de los recursos ha de ser considerado por su especificidad, esto es, debe ser concretado sobre el campo de investigación, al tiempo que debe ser considerado en su carácter relacional.

Los recursos tienen carácter relacional, en el sentido de que los agentes y los recursos se encuentran en un continuo proceso de re-definición. Los recursos no están dotados de intencionalidad ni de finalidad por sí mismas, sino que son dotadas de estas cualidades por efecto de la interacción social de los diferentes agentes en estructuras sociales, o lo que es lo mismo por su carácter relacional y conflictivo. Giddens (2009) sostiene que el poder presupone relaciones de autonomía y de dependencia entre actores en contexto de interacción:

Todas las formas de dependencia ofrecen algunos recursos por medios de los cuales aquellos que son subordinados pueden influir en las actividades de sus superiores. Es eso que llamo de dialéctica del control en sistemas sociales (GIDDENS, 2009, p. 19). 
Los agentes consideran los recursos como instrumentos para el desarrollo de acciones de transcendencia espacial. El análisis de estas acciones debe ser realizado conjuntamente con el análisis de los recursos puestos en juego. La cuestión esencial es que debemos diferenciar los recursos fundamentales, que están en el centro de las relaciones de conflicto y/o cooperación, de los recursos diferenciales, esto es, aquellos que permiten establecer diferencia entre unos agentes y otros, debido a la posibilidad que tiene de otorgarle un uso diferenciado y no controlado por los otros agentes. En este sentido, podemos diferenciar los recursos de las instituciones públicas de planificar e construir espacios de uso turístico, del uso que hacen los residentes y/o turistas, que pueden ser distintos a los planificados, según esa dialéctica del control sugerida por Giddens (2009).

Los medios o recursos para la realización de actividades de transcendencia espacial están determinados por las distintas lógicas de cada uno de los agentes. Así considerada, la disponibilidad de recurso es a priori infinita. Pablos y colaboradores (1999) consideran que "cualquier cosa que pueda utilizarse con carácter instrumental es un recurso" (PABLOS, et. al. 1999, p. 62). Conviene precisar que nos referimos a 'cualquier cosa', de la misma forma como lo emplea I. Illich, para referirse a herramientas:

Claramente yo empleo el término herramienta en el sentido más amplio posible, como instrumento o como medio, independientemente de ser producto de la actividad fabricadora, organizadora o racionalizante del hombre, (...) es decir, para ser puesto al servicio de una intencionalidad (ILLICH, 1974, p. 41). 
Un grupo geográfico-social de comportamiento es un agente cuando dispone de recursos. Un elemento material o simbólico es un recurso cuando un agente lo utiliza para desarrollar actividades de transcendencia espacial. El fenómeno del turismo es un conjunto de realidades múltiples en la cual la diversidad de agentes no necesariamente se relaciona con todos los otros agentes y cada uno de ellos realiza una atribución de sentido diferente sobre los recursos disponibles. De esta forma, necesitamos especificar los recursos fundamentales, los recursos diferenciales que está siendo disputado y los agentes que desarrollan actividades de transcendencia espacial para cada parcela de actividad de forma específica y de modo relacional. Así, podemos percibir que los recursos utilizados por las instituciones internacionales, las instituciones políticas, los movimientos sociales, los empresarios, los residentes y los turistas no sólo son de naturaleza distinta, sino que son utilizados en parcelas distintas de la realidad, de acuerdo con la racionalidad limitada, la reflexividad estructural y autoreflexividad de cada uno de los agentes.

\section{Consideraciones finales}

En este capítulo hemos expuesto algunas reflexiones para analizar el turismo en la sociedad contemporánea de forma que evite el análisis unidimensional. El turismo es un fenómeno complejo que contribuye a la re-construcción de la sociedad, a partir de las acciones de los agentes que construyen las formas espaciales (dimensión material) y las identidades locales (dimensión simbólica). La diversidad de agentes (complejidad sistémica) construyen la realidad 
desde distintas interpretaciones (complejidad fenoménica), estableciendo relaciones de cooperación y conflicto en relación a los distintos recursos existentes.

Los agentes realizan sus actividades de transcendencia espacial en distintas parcelas de la realidad (realidades múltiples), con interpretaciones y recursos diferenciados. Entender las lógicas de cada uno de los agentes (racionalidad limitada) es de vital importancia para la comprensión del fenómeno del turismo. Las distintas formas de interpretar la realidad orientan las preferencias espaciales y las acciones de transcendencia espacial. El espacio turístico construido es el resultado de las acciones de todos los agentes que es diferente a la intencionalidad de cada uno de ellos analizados aisladamente.

El sistema de lugares funcionales de uso turístico tiene que ser entendido como un sistema dinámico, en el que los agentes participan en contextos estructurados y reflexivos. Los agentes son reflexivos, tanto sobre las reglas y recursos, como sobre los otros agentes y su propia posición en el contexto de la acción. Esta reflexividad impide que el análisis de la re-construcción material y simbólica del turismo pueda ofrecer una conclusión definitiva, dado que continuamente recurso, reglas y agentes se encuentran en un proceso de re-definición constante debido al carácter relacional de dicho fenómeno.

La aplicación de esta reflexión requiere un proceso de traducción en cada realidad turística de forma específica, debido al carácter dinámico de la sociedad y de la teoría social. La finalidad es ofrecer una visión más compleja de la realidad integrando autores de diferentes disciplinas académicas 
(antropología, filosofía, geografía, historia, sociología y turismo) que ofrecen distintas formas de analizar el turismo. En definitiva, el trabajo de los investigadores del turismo participa del proceso de re-construcción del fenómeno del turismo aportando una interpretación que debe ser relacional y reflexiva.

\section{Referencias}

BAUMAN, Zygmunt. La cultura como praxis. Barcelona: Paidós, 2002.

BAUMAN, Zygmunt. Modernidad y ambivalencia. IN: BERIAN, Josetxo (Comp.). Las consecuencias perversas de la modernidad. Barcelona: Anthropos, 1996.

BECK, Ulrich. O que é globalização. Equivocos do globalismo, respostas à globalização. São Paulo: Paz e Terra, 1999.

BERGUER, Peter, LUCKMANN, Thomas. La construcción social de la realidad, Madrid: Amorrortu, 1994.

CANCLINI, Nestor. Culturas híbridas. Estratégias para entrar e sair da modernidade. São Paulo: EDUSP, 2006.

CASTELLS, Manuel. La era de información. La sociedad red. Madrid: Alianza Editorial, 2005.

CORIOLANO, Luiza Neide. Turismo e geografía. Abordagens Críticas. Fortaleza: Editora Universidade de Ceará, 2005. 
DAHRENDORF, Ralf. Las clases sociales y sus conflictos en la sociedad industrial. Madrid: Rialp, 1979.

EINSTEIN, Albert. Mi visión del mundo. Barcelona: Tusquet, 1986.

FEATHERSTONE, Michael. Localismo, globalismo e identidade cultural. Revista Sociedade e Cultura, v. 11, n. 1, Janeiro/Junho, 1996.

FEYERABEND Paul. Contra el método. Hacia una teoría anarquista del conocimiento. Barcelona: Planeta Agostini, 1993.

GIDDENS, Anthony. A constituição da sociedade. São Paulo: Martins Fontes, 2009.

HABERMAS, Jünger. La lógica de las ciencias sociales. Madrid: Tecnos, 1996.

HALL, Stuart. A identidade cultural na pós-modernidade. Rio de Janeiro: DP\&A Editora, 2006.

HARVEY, David. A construção capitalista do espaço. São Paulo: Annablume, 2006.

HIERNAUX, Daniel. La fuerza de lo efímero. Apuntes para la construcción de la vida cotidiana en el turismo. IN: LINDON, Alicia: La vida cotidiana y su espacio-temporalidad. Barcelona: Anthropos, 2000.

HOSBAWN, Eric. A era dos impérios. 1875-1947. São Paulo: Paz e Terra, 2005. 
ILLICH, Ivan. La convivencialidad. Barcelona: Barral, 1974.

KRIPPENDORF, Jost. Sociologia do Turismo. São Paulo: Aleph, 2003.

LASH, Scott. La reflexividad e sus dobles: estructura, estética, comunidad. IN: BECK, Ulrich; GIDDENS, Anthony; LASH, Scott: Modernidad reflexiva. Política, tradición y estética en el orden social moderno. Madrid: Alianza, 1994.

LEFEBVRE, Henri. Espacio y política. El derecho a la ciudad II. Barcelona: Península, 1976.

LUHMANN, Niklas. Sociedad y sistema. La ambición de la teoría. Barcelona: Paidós, 1997.

MARCH, J. G.; SIMON, H.A. Los límites cognitivos de la racionalidad. IN: RAMIÓ, Carles; BALLART, Xavier, Lecturas de teoría de la organización. Madrid: Ministerio para las Administraciones Públicas, 1993.

MAIER, Jörg; PAESLER, Reinhard; RUPPERT, Karl; SCHAFFER, Franz. Geografía social. Madrid: Rialp, 1987.

MARX, Karl. ENGELS, Federic. El manifiesto comunista. Madrid: Editorial Ayuso, 1977.

BOYER, Marc. História do turismo de massas. Bauru-São Paulo: EDUSC, 2003.

MEDINA, Julio César. Re-construcción material y simbólica del espacio urbano: el Albayzín de Granada, 
Patrimonio de la Humanidad. Granada: Editora de la Universidad de Granada, 2009.

MEDINA, Julio César. "Circularidad dialógica entre los significados y los números", IN: DINIZ, Ariosvaldo; BRASILEIRO, Maria Dilma; LATIESA, Margarita (Org.). Cartografias das novas investigações em sociologia. João Pessoa: EDU-UFPB/Manufatura, 2005.

MORIN, Edgar. Introducción al pensamiento complejo. Barcelona: Gedisa, 1996.

PABLOS, Juan Carlos; Pascual, Nuria; Gómez, Yago. El domino de lo cotidiano: la búsqueda de la calidad de vida, Revista Española de Investigaciones Sociológicas, Madrid, n. 86, abril-junio, 1999.

PABLOS, Juan Carlos de; MEDINA, Julio César. Las lógicas del turismo: del consumo a la construcción de la sociedad. IN: BLANQUER, David: Turismo cultural y urbano. Valencia (España): Tirant lo Blanch, 2004.

SANTOS, Milton. Pensando o Espaço do Homen. São Paulo: Editora EDUSP, 2007.

WALLERSTEIN, Inmanuel. A estruturação capitalista e o sistema-mundo, IN: GENTILI, Paulo (org.), Globalização excludente. Desigualdade, exclusão e democracia na nova ordem mundial. Petrópolis: RJ. Vozes, 2002.

WEBER, Max. La ética protestante y el espíritu del capitalismo. Madrid: Albor, 1998 
